\title{
Práctica pedagógica, dominio afectivo y procesos matemáticos de los docentes de matemáticas en el nivel de educación básica del sector publico.
}

\section{Pedagogical practices, affective domain and mathematical processes of mathematics' teachers in basic education of the establishments of the public sector}

\author{
a Julio Andrés Niño-Blanco, ${ }^{\mathrm{b} C e s a r}$ Augusto Hernández-Suárez, ${ }^{\mathrm{T}}$ Marcia Yelitza Bonilla- González \\ $\mathrm{a}^{*}$ Magister en Educación Matemática, Docente Institución Educativa Colegio Comfanorte, Los Patios - Colombia, julioandresnb@ufps.edu.co, \\ Orcid: 0000-0002-6826-8789 \\ ${ }^{\mathrm{b}}$ Magister en Enseñanza de la Ciencias Básicas mención Matemática, Docente Universidad Francisco de Paula Santander Cúcuta -Colombia, \\ cesaraugusto@ufps.edu.co, Orcid: 0000-0002-7974-5560 \\ ${ }^{c}$ Magister en Lingüística Aplicada, Docente Universidad Santo Tomas, Cúcuta - Colombia, marciabonilla@ustadistancia.edu.co, Orcid: 0000-0001- \\ $8402-5317$
}

Forma de citar: Niño-Blanco, J. A. Hernández-Suárez, C.A. Bonilla- González, M.Y Práctica pedagógica, dominio afectivo y procesos matemáticos de los docentes de matemáticas en el nivel de educación básica del sector publico. Eco Matemático, 10 (1), 19-27

Recibido: 17 agosto 2018

Aceptado: 2 noviembre 2018

\section{Palabras clave \\ prácticas \\ pedagógicas, \\ lineamientos \\ curriculares, \\ estándares \\ curriculares, \\ dominio afectivo, \\ procesos \\ matemáticos.}

Resumen: El estudio de las prácticas pedagógicas ha permitido contribuir al mejoramiento de los docentes en el aula de clase teniendo en cuenta los lineamientos propuestos por el ministerio de educación. Sin embargo, la realidad es otra debido a que estas mejoras se han quedado en teorías y no han promovido un cambio significativo en la manera de actuar de los mismos. El presente artículo es el resultado de una investigación el cual tuvo como objetivo analizar las prácticas pedagógicas y dominio afectivo sobre la enseñanza de la matemática en docentes de educación básica, y en particular indagar sobre la presencia de los procesos matemáticos propuestos por el Ministerio de Educación Nacional de Colombia. Con los participantes, quienes fueron docentes de área de matemáticas, se implementó una entrevista semiestructurada, instrumentos propios de un método cualitativo con enfoque hermenéutico. Para el análisis, el autor aplicó la técnica de codificación y uso del software Atlas-ti a fin de crear confiabilidad y validez en el mismo. Los resultados de los instrumentos fueron comparados con los lineamientos y estándares curriculares del área de matemáticas propuestos por el Ministerio de Educación Nacional. A partir de lo anterior, se concluyó que los docentes a pesar que emplean cierta documentación de la propuesta ministerial, necesita innovar sus prácticas pedagógicas teniendo en cuenta la propuesta curricular fundamentada en la propuesta ministerial.

\section{Keywords}

pedagogical practices, curricular guidelines, curricular standards,

\begin{abstract}
The study of pedagogical practices have allowed to contribute to the teachers' improvement in the classroom by taking into account the guidelines proposed by the Ministry of Education. However, the reality in different because these improvement have remained in the theories and has not promoted a meaningful change in the way they act. This article is the result of an investigation which had as objective to analyze the pedagogical practices and affective domain on the teaching
\end{abstract}

*Autor para correspondencia julioandresnb@ufps.edu.co

http://dx.doi.org/10.1016/j.eq/

2590-9215@ 2017 Universidad Francisco de Paula Santander. Este es un artículo bajo la licencia CCBY 


\section{Keywords}

affective domain, mathematical process. of mathematics in teachers of basic education, and in particular to investigate on the presence of the mathematical processes proposed by the Ministry of National Education of Colombia. The participants who were teachers of mathematics participated in semistructured interview. These instruments were applied focused on the qualitative method and the hermeneutics such as research approach. For the analysis, the author applied the codification and Atlas.ti software such as technique in order to create reliability and validity in this paper. The instrument results were compared with the guidelines and mathematics curricular standards proposed by the Ministry of Education. Bearing in mind above, the researcher inferred that although the teachers used some information from the Ministry of Education, they might need to innovate the pedagogical practices taking into account the curriculum gave by the ministry.

\section{Introducción}

Es indiscutible la preocupación de los docentes con respecto a la calidad de la educación y a su constante cambio ya que como se evidencia, la sociedad actual exige una educación que sea capaz de responder a los retos y necesidades que presentan los estudiantes, por lo que el desempeño de la labor docente sebe ser considerado como un factor primordial en la formación y desarrollo de la sociedad (Parada, 2018). No obstante, dichos cambios han influido en cómo los docentes diseñan, ejecutan y evalúan sus prácticas pedagógicas lo cual hace evidente la relación entre el currículo y la manera como los maestros lo desarrollan y lo mejoran en la realización del trabajo. Según Jiménez, Limas y Alarcón (2016, p. 131) la intervención del profesor en el aula es un auténtico proceso de investigación, puesto que requiere diagnosticar los diferentes estados y movimientos de la compleja vida del aula, desde la perspectiva de quienes intervienen en ella, y elaboran, experimentan, evalúan y redefine los modos de intervención en virtud de los principios educativos que justifican y validan la práctica tanto la propia evolución individual como la colectiva de los estudiantes. Es decir, el maestro es quien orienta el proceso de enseñanza-aprendizaje y busca estrategias para desarrollar sus actividades teniendo en cuenta las necesidades de cada población.

Con base en lo expuesto, en los últimos años, el Ministerio de Educación Nacional (en adelante MEN) de Colombia ha estado modificando y mejorando la metodología de las prácticas pedagógicas de los docentes a nivel nacional con el fin de favorecer la enseñanza en el proceso educativo. Dichas mejoras se han enfocado en responder las exigencias del contexto educativo a través de estándares y proyectos curriculares. De la misma manera, la propuesta busca fortalecer las habilidades de los estudiantes con el fin de generar ciudadanos competentes e integrales capaces de desenvolverse en la sociedad. Según el MEN (2014) el uso de estándares y lineamientos permiten monitorear los avances en el tiempo y diseñar estrategias focalizadas de mejoramiento acorde con las necesidades de las regiones e instituciones educativas.

No obstante, estas directrices propuestas a nivel educativo no son suficiente para lograr la meta del Ministerio de Educación, sino que también se hace indispensable el compromiso porparte de los docentes sobre su quehacer pedagógico y su implementación de forma organizada y coherente. Dando como resultado un mejoramiento en las competencias de los estudiantes por medio de la articulación de los lineamientos y los estándares, especialmente en el área de matemáticas.

Partiendo de lo anterior, se necesita reflexionar sobre la definición de las prácticas pedagógicas y su influencia en el proceso de enseñanza-aprendizaje. Según Barreto y Mejía (2015, p 89) "la práctica pedagógica busca interpretar y describir los patrones

"las representaciones semióticas son a la vez representaciones conscientes y externas... permiten una mirada del objeto a través de la percepción de estímulos (puntos, trazos, caracteres, sonidos...) que tienen el valor de significantes" (Duval, 2017: 68). las figuras geométricas, los gráficos cartesianos, los esquemas, la escritura aritmética y algebraica, las tablas son algunas de las representaciones semióticas de mayor uso en la enseñanza y el aprendizaje de la geometría 
de las creencias y la interacción entre maestros y alumnos durante las mismas". El maestro como agente activo en el proceso educativo, debe orientar al estudiante en la búsqueda de conocimiento por medio de técnicas de enseñanza, sin olvidar la relación cordial y amable que se debe tener en el aula de clase.

Igualmente, Castro, Peley y Morillo (2006) consideran la práctica pedagógica como una acción que permite innovar, profundizar y transformar el proceso de enseñanza del docente en el aula. Es decir, el profesor es el responsable en el desarrollo de las competencias de losestudiantes tanto en el salón de clase como en su formación personal fuera de la misma.

Por su parte, Jiménez, Limas y Alarcón (2016, p. 135) define la práctica pedagógica como:

[...] actividad intencionada, cotidiana y recursiva, que se despliega en determinados marcos institucionales. Recursiva por la repetición de las acciones en condiciones más o menos semejantes. La cotidianidad se presenta dentro de un marco institucional en el que inciden aspectos de naturaleza política como el currículum y diversas demandas sociales. La práctica es una forma de poder e institucionalidad que actúa tanto a favor del cambio como de la continuidad; es decir que otorga a los profesores márgenes de posibilidades para el cambio y la transformación.

En otras palabras, las prácticas pedagógicas son actividades realizadas por el docente conel fin de que el estudiante se convierta en un agente activo y dinámico en su propio procesoacadémico, es decir, el estudiante no debe ser en un receptor pasivo y limitante incapaz dereflexionar, interpretar y aplicar su conocimiento.

De esta forma es interesante analizar las prácticas como como un proceso dinámico, donde eldocente es autónomo de implementar actividades acorde a las necesidades y temáticas del salón de clase; pues como lo señala Vasco (1990) las prácticas pedagógicas es un proceso de enseñanzaaprendizaje, requiere de la intersubjetividad de los frentes partícipes, es decir, de la relación entre teoría - práctica, docente - conocimiento, docente - estudiante, ambiente de aprendizaje - efectividad del conocimiento, etc.

Por otra parte, es importante mencionar algunos aspectos que conlleva unas buenas prácticas pedagógicas ya que influyen en el proceso de enseñanza y aprendizaje de los estudiantes en el área de matemáticas. En el presente artículo se destacan los aspectos relacionados con las creencias y actitudes, así como la presencia de procesos matemáticos en docentes. Fernández, Hernández, Prada y Ramírez (2018) hacen una revisión sobre el dominio afectivo y las prácticas pedagógicas de docentes de Matemáticas. Por otro parte, Gómezescobar, León y Fernández (2019), realizan un estudio exploratorio en maestros desde las actitudes hacia las matemáticas y la práctica docente. Aunque el manuscrito se centra en los docentes, en cuanto a estudiantes, se pueden mencionar los trabajos de Fúneme y Hernández (2017), relacionado identificación de procesos matemáticos en la comprensión de conceptos en estudiantes universitarios y el de Duarte, Ricardo y Santos (2018) sobre el dominio afectivo hacia las matemáticas en estudiantes de educación media.

Ramos y Casas (2018) establecen que las creencias son ideas poco elaboradas que forman parte del conocimiento que posee una persona e influyen de manera directa en su desempeño. Las creencias en el ámbito educativo inciden, de manera decisiva, en todo lo que supone el proceso de enseñanza aprendizaje.

También se acepta, de acuerdo con Gómez (2010) que las creencias, además del componente cognitivo, pueden tener un componente de tipo afectivo. Además, es necesario distinguir las estructuras de creencias individuales de los sistemas 
de creencias social o culturalmente compartidas. En tal sentido, se concibe que las creencias conforman un conjunto de opiniones del individuo que se refuerzan y apoyan mutuamente, que son primordialmente de tipo cognitivo pero que, a menudo, incorporan lo afectivo.

Con respecto a la actitud, Vázquez y Manassero (2007, p.2) la definen como la forma expresiva del ánimo que tenga un individuo en relación con la acción que se tome en las situaciones que se le presente en momentos determinados. A veces se tiene una visión muy reducida de la actitud, como una predisposición positiva o negativa limitada al aprendizaje.

Finalmente, Allport (1973) menciona que las capacidades del ser humano pasan por la organización de la personalidad y que la personalidad misma representa un todo integral que incluye en una unidad funcional todos los diversos sistemas de comportamiento que posee un individuo. De tal modo, que las aptitudes forman un cuadro integral donde los seres humanos son aptos para unas cosas y no para otras.

En este marco de ideas, las aptitudes para las matemáticas, probablemente, están referidas según Valiente (2000), a la resolución de problemas matemáticos y a diversas actividades donde se utilice las operaciones de adición, sustracción, multiplicación y división como operaciones básicas para que el estudiante se desenvuelva eficientemente en la vida. De esta manera, el uso de los procesos matemáticos permite fomentar en los estudiantes el dominio de las habilidades y estrategias que les permitan aprender a aprender, así como la utilización de los conocimientos disponibles para dar respuesta a situaciones cambiantes y distintas.

En investigaciones se han encontrado el uso de las prácticas pedagógicas y su influencia en la matemática, encontrando aspectos relevantes, Enamorado (2012) en su investigación tiene como propósito conocer las prácticas pedagógicas de los docentes de básica primaria en el área de matemáticas y su influencia con los resultados de las pruebas externas. Este estudio se abordó desde un enfoque cualitativo teniendo por diseñó un método no experimental. Igualmente, Rodríguez y Herrera (2015) mediante una investigación bajo el enfoque cualitativo de tipo de descriptivo. Este estudio analizó la relación entre los lineamientos curriculares dadas por el Ministerio de Educación y las prácticas pedagógicas que realizan los docentes en el aula de clase en el cual concluyeron que los docentes de matemáticas desconocen en gran parte la teoría de dicha propuesta, y quienes la conocen a la hora de desarrollar las prácticas pedagógicas en clase no aplican los lineamientos o estándares curriculares y emplean prácticas que dejan por fuera herramientas sugeridas por el MEN.

\section{Materiales y métodos}

Para la investigación se adoptó la hermenéutica como tipo de investigación ya que permite la interpretación de las opiniones dadas por los participantes. Así mismo, este método ayuda a indagar y analizar el discurso permanentemente. Según Rueda y Villaroel (1992), el método hermenéutico la define como el proceso por medio del cual conocemos la vida psíquica la ayuda de signos sensibles que son su manifestación. En otras palabras, es el arte de la interpretación de las palabras tanto escrito como oral, pero conservando su contexto el cual forma parte.

Igualmente, la investigación diseñó una estructura en el cual toma las prácticas pedagógicas como el objeto de estudio que corresponde al todo y en las temáticas que la conforman como sus partes. Sin embargo, es importante aclarar que durante la aplicación del círculo hermenéutico es posible modificar dichas temáticas al proceso investigativo.

La estructura diseñada está dividida en cinco partes fundamentales. La primera es la indagación en la cual se centra en la creación de preguntas 
del tema investigativo. La segunda se enfoca en estudio de textos y autores referentes a la pregunta investigativa realizada anteriormente. La tercera fase se enfoca en la lectura y diálogo con los textos y autores que intervienen en la investigación. La cuarta fase se centra es la comparación entre la literatura revisada y la práctica generada en el presente estudio y finalmente la última fase se basa en la interpretación y comprensión de los discursos adquiridos. Cada una de las partes permite analizar detalladamente las variables que influyen en la presente investigación. Así mismo, ayuda a sintetizar el producto final de la investigación teniendo en cuenta el proceso realizado anteriormente y los resultados de los instrumentos de la investigación.

Basado en este tipo de investigación, se seleccionó el método cualitativo descriptivo en este estudio con el fin de obtener información confiable y verídica a través del contexto haciendo uso de los diferentes instrumentos de recolección de información (encuestas, entrevistas, grabación entre otros) además del análisis de información. De acuerdo con Taylor y Bogdan (1986, p. 20) consideran la investigación cualitativa como aquella que produce datos descriptivos: las propias palabras de las personas habladas o escritas y la conducta observable. Los instrumentos utilizados fueron encuesta, entrevista, grabación y software Atlas-Ti. Cada uno ayudo en el análisis de resultados.

Los participantes seleccionados en el presente estudio son docentes del área de matemáticas de básica primaria en los tres establecimientos educativos del sector público. Todos los docentes están nombrados en propiedad por el MEN con una amplia experiencia. Los participantes fueron seleccionados en dos diferentes grupos. El primer grupo fue integrado por 53 participantes en diferentes rangos de edad y género. El segundo grupo se seleccionó de acuerdo al resultado del primer grupo y está conformado por seis participantes. El área de investigación corresponde a los tres (3) establecimientos educativos públicos del Municipio de los Patios, Norte de Santander, para lo cual se entrevistó dos (2) docentes de cada una de ellas: Instituto Técnico Mario Pezzoti, Colegio Once de Noviembre y Colegio Patio Centro N².

\section{Resultados y Análisis}

Para el análisis e interpretación de la información que proporcionaron los participantes del proceso, la cual se recolectó a través de una entrevista en profundidad, fue de tipo cualitativo, el cual da cuenta del proceso de sistematización lógica y coherente de los hallazgos encontrados buscando una comprensión más profunda de lo que se ha estudiado refinando las interpretaciones, a través del análisis de contenido, definido según Pérez (2000), como un procedimiento para la categorización de datos verbales y de conducta con fines de clasificación, resumen y tabulación (p. 134). Por otra parte, se presentan algunos comentarios al respecto de las categorías, en la misma de aquí en adelante cuando aparezca [3:7] corresponderá al documento " 3 ", segmento de información " 7 ", con la finalidad de identificar rápidamente los comentarios.

Este análisis fue dividido en tres dimensiones. Cada dimensión se categorizó con códigos teniendo en cuenta sus indicadores. La primera dimensión el cual se categorizó (ver Tabla 1) son las creencias en el cual se obtuvieron los siguientes resultados: algunos de los docentes entrevistados expresaron que es una ciencia difícil de aprender, que debe estar destinada a personas inteligentes, la cual genera temor en los estudiantes. Esto hace ver que los docentes, relacionan el aprendizaje de la matemática como algo complejo, pero también afirman que el papel del docente es muy importante para motivar a los estudiantes en esta noble tarea.

A continuación, se presentan algunos de los comentarios "los estudiantes creen que el área de matemáticas es solamente para personas de muy alta inteligencia...allí están errados" [7:3]. De la misma manera, es importante señalar que las creencias de los profesores son muy importantes dentro de 
la práctica pedagógica, específicamente en las actividades de enseñanza. Al respecto, los profesores encuestados manifiestan que los estudiantes creen que las matemáticas son difíciles, y que eso influye en el aprendizaje de estas, asimismo en el rendimiento académico sin olvidar que disminuye la confianza de los educandos, lo cual incide en el trabajo dentro del aula. Tal como lo muestra el siguiente comentario; "los estudiantes ya que es una ciencia pura y exacta si piensan que son difíciles, estas creencias condicionan para que el aprendizaje se lleve a cabo" [9:3].

Según lo anterior, los docentes manifiestan que las prácticas pedagógicas deben tener en cuenta las teorías educativas, la percepción y los valores de los docentes sin olvidar la descripción y el pensamiento del mismo ya que tiene que ver con el saber(pedagógico) adquirido en el proceso de formación, así como las experiencias vividas (saber práctico), los cuales tienen una influencia directa en las habilidades que va desarrollando el docente de matemáticas para desempeñar diferentes actividades asociadas con la práctica pedagógica.

Tabla I.Síntesis de los códigos, indicadores, dimensión creencias en la categoría: prácticapedagógica del profesor de matemática.

\begin{tabular}{|c|c|c|c|}
\hline Códigos & Indicador & Dimensiones & Categoría \\
\hline Modelo de enseñanza & \multirow{3}{*}{ Concepto de creencia } & \multirow{8}{*}{ Creencias } & \multirow{8}{*}{$\begin{array}{l}\text { Las prácticas } \\
\text { pedagógicas }\end{array}$} \\
\hline Método & & & \\
\hline Rechazo & & & \\
\hline Saber pedagógicos & \multirow{3}{*}{$\begin{array}{l}\text { Relación concepto- } \\
\text { creencia }\end{array}$} & & \\
\hline Saber didáctico & & & \\
\hline Saber práctico & & & \\
\hline Motivación & \multirow{2}{*}{$\begin{array}{c}\text { Influencia de las } \\
\text { creencias en la } \\
\text { enseñanza }\end{array}$} & & \\
\hline Rendimiento académico & & & \\
\hline
\end{tabular}

A continuación, se presenta la organización de los datos primarios por categoría, dimensiones, indicador y códigos. En este sentido, se muestra la organización de la Dimensión Actitudes, para su análisis se utilizaron los indicadores: a) Actitud del docente hacia la asignatura, b) Actitud del estudiante frente a la asignatura y su influencia desde la perspectiva docente (Ver Tabla 2)
Tabla II. Síntesis de los códigos, indicadores, dimensión actitudes en la categoría: práctica pedagógica del profesor de matemática.

\begin{tabular}{|c|c|c|c|}
\hline Códigos & Indicador & Dimensiones & Categoría \\
\hline $\begin{array}{l}\text { Acciones didácticas } \\
\text { pedagógicas }\end{array}$ & \multirow{3}{*}{$\begin{array}{l}\text { Actitud del docente } \\
\text { hacia la asignatura }\end{array}$} & \multirow{6}{*}{ Actitudes } & \multirow{6}{*}{$\begin{array}{l}\text { Las prácticas } \\
\text { pedagógicas }\end{array}$} \\
\hline Habilidades y destrezas & & & \\
\hline Manejo de emociones & & & \\
\hline Pensamientos & \multirow{3}{*}{$\begin{array}{l}\text { Actitud del estudiante } \\
\text { frente a la asignatura y } \\
\text { su influencia desde la } \\
\text { perspectiva docente }\end{array}$} & & \\
\hline Expresiones & & & \\
\hline Emociones & & & \\
\hline
\end{tabular}

Los profesores en la entrevista relacionaron la actitud con emociones, estados de ánimo, métodos, canales de comunicación, que de ser efectivas influyen en el desarrollo de competencias de los estudiantes. Igualmente, según las opiniones de los participantes, los docentes deben manejar sus emociones y mostrar seguridad en el aula de clase con el fin de generar confianza en el estudiante y a su vez un óptimo proceso de aprendizaje. Tal como lo expresa el siguiente comentario "un manejo adecuado de las emociones....pues lo que hace puede representar la diferencia entre un alumno con potencial y otro con poca motivación que derive en fracaso escolar.” [9:6].

Es importante señalar que referente al indicador b) Actitud del estudiante frente a la asignatura y su influencia desde la perspectiva docente, evidentemente la actitud, siendo una predisposición positiva o negativa frente a algo, cabe destacar que cuando se refiere a un área, especialmente Matemática, esto incide en el aprendizaje. Al respecto, los docentes entrevistados manifestaron que la actitud de los estudiantes hacia la matemática es apática, se muestran desmotivados, desinteresados. Sin embargo, el papel del docente es muy importante para que el estudiante deje las falsas creencias y predisposición negativa hacia la matemática. En este orden de ideas, se presentan algunos de los comentarios "las creencias más comunes es la apatía, el no pensar, no me gusta esa área...no la entiendo, no soy capaz...es muy difícil," [9:7]. la dimensión actitudes hacia la matemática, está relacionada con la parte afectiva de los docentes, así como la de los estudiantes, manifestada en deseos, voluntad y sentimientos acerca de algo, y 
se exteriorizan en las actuaciones, tiene que ver con la disposición, convicción y creencias de los diferentes actores frente a la enseñanza aprendizaje de la Matemática.

La tercera dimensión se enfoca en los procesos matemáticos en el cual se presenta la organización de los datos primarios por categoría, dimensiones, indicador y códigos. En este sentido, se utilizaron los indicadores: a) Desarrollo de las competencias, b) Resolución de problemas, c) Razonamiento, d) Conexiones y e) Representación. (Ver Tabla 3)

La Matemática es una ciencia amplia, además de compleja, tiene múltiples interrelaciones con todas las áreas del saber, se convierte a su vez en un medio que utilizan las personas para el desarrollo personal, intelectual así como también la construcción de conocimiento. Rodríguez (2015) afirma que las matemáticas contribuyen al mantenimiento $\mathrm{y}$ desarrollo de ideologías, forman parte de nuestros sistemas de creencias. Por esto, la importancia de desarrollar estudiantes competentes en el área del saber, pues esta abre nuevas oportunidades en el ámbito laboral y la transformación del medio que le rodea. Los profesores entrevistados opinaron que las competencias son habilidades y operaciones que están entrelazadas, las cuales permiten pensar, comunicar y resolver situaciones matemáticas. A continuación, se presentan comentarios que evidencian la dimensión: Procesos matemáticos; "La verdad es que entre las competencias todas estas entrelazadas, pero creo yo que la de mayor esfuerzo en el aula de clase requiere es resolución de problemas y razonamiento" [7:12]. Además, los docentes entrevistados manifiestan que la resolución de problemas permite que los estudiantes aprendan haciendo, y entre las estrategias que utilizan se encuentran las lúdicas, además de situaciones de la vida cotidiana tal como lo muestra el siguiente comentario "Yo aplico ensayo-error, comparación, aplicaciones en la vida diaria, aprender haciendo" [3:10]. Ahora bien otro de los indicadores que definen los procesos matemáticos es el Razonamiento porque permite que los estudiantes interpreten la realidad considerando los conocimientos matemáticos previos. En este sentido, los docentes opinaron "Desarrollo de problemas, de aplicación de la vida diaria... aprender haciendo que sean ellos mismos que propongan y solucionen problemas" [3:11].

De esto se infiere, que los procesos permiten que los estudiantes comprendan los conocimientos matemáticos, y puedan llevarlo a las situaciones de la vida diaria, permitiendoresolver problemas, por mediodelacombinacióndediferentesprocedimientos tales como conexión, representación, resolución de problemas entre otros, entendiéndose esta como las habilidades y conocimientos integrados para resolver situaciones problemáticas de la vida.

Tabla III. Síntesis de los códigos, indicadores, dimensión procesos matemáticos en la categoría: práctica pedagógica del profesor de matemática.

\begin{tabular}{lllll}
\hline \multicolumn{1}{c}{ Códigos } & \multicolumn{1}{c}{ Indicador } & Dimensiones & Categoría \\
\hline Estrategias del docente & $\begin{array}{l}\text { Desarrollo de } \\
\text { competencias }\end{array}$ & las & & \\
\cline { 1 - 2 } Saber pedagógico & $\begin{array}{l}\text { Resolución } \\
\text { problemas }\end{array}$ & de & $\begin{array}{c}\text { Procesos } \\
\text { matemáticos }\end{array}$ & $\begin{array}{l}\text { Las prácticas } \\
\text { pedagógicas }\end{array}$ \\
\cline { 1 - 2 } Tareas matemáticas & Razonamiento & & & \\
\hline Vida cotidiana & Conexiones & & & \\
\hline Interpretar & Representación & & & \\
\hline Sincular, relacionar & Simbolizar & &
\end{tabular}

\section{Conclusiones}

Después de la realización del estudio y el análisis de las prácticas pedagógicas de los docentes en el área de matemáticas de las tres instituciones educativas del municipio de los Patios, por medio de la aplicación de la encuesta y entrevista semiestructurada a los docentes, la interpretación y categorización de la información, se obtuvo una serie de resultados el cual permitió que el investigador sacara las siguientes conclusiones.

Se determinó la concordancia entre la propuesta teórico - pedagógica del MEN y las prácticas pedagógicas donde se encontraron que los docentes de matemáticas emplean los documentos otorgados por el MEN en sus planes de aula y asignatura. Así mismo, los educadores conocen la propuesta ministerial y tratan de integrarlas en su práctica pedagógica de acuerdo a las necesidades 
que presente el grupo de estudiantes en general, ya que como los profesores mencionan, en el aula de clase influyen muchos elementos externos que hace el proceso de enseñanza-aprendizaje más complejo.

Los docentes se esfuerzan por lograr un aprendizaje significativo en los estudiantes en el área de matemáticas, independientemente si es su perfil profesional o no. Por ello, ellos realizan diferentes actividades que sean atractivas por los estudiantes y así enriquecer su conocimiento, por ejemplo; se pudo observar que los educadores emplean situaciones de la vida real o áreas de interés de los estudiantes para integrar la matemática, tales como la artística o el deporte. Esto con el fin de dejar el uso del cuaderno como centro de estudio de las diferentes temáticas.

En cuanto a las creencias, actitudes y manejo de los procesos matemáticos que tienen los docentes, se puede deducir que los educadores es un elemento importante en el proceso de enseñanza-aprendizaje, por lo tanto sus actitudes y creencias influyen en la manera como el estudiante percibe la asignatura. De acuerdo a los entrevistadores, las creencias pueden ser positivo o limitantes para el aprendizaje de un área en particular el cual influye en las motivaciones de un estudiante por aprender. Por lo tanto, un docente debe manejar adecuadamente sus emociones puesto que puede presentar la diferencia entre un estudiante con potencial y otro con poca motivación que propicie en fracaso escolar.

En general, en cuanto al diseño temático de la clase y el manejo de las creencias, actitudes y procesos matemáticos, los docentes evidencian conocimiento no sólo frente a la asignatura de la matemática, sino además de los factores que influyen el proceso de enseñanza-aprendizaje tales como la actitud del estudiante en el aula y la respuesta del docente en el aula. Si bien es cierto, que los procesos matemáticos son indispensables para la preparación de unas pruebas externas, también lo es la actitud del estudiante para aprender.
Finalmente, se puede concluir que la mayoría de los docentes emplean los documentos emitidos por el Ministerio de Educación en su plan de aula. Sin embargo, al aplicarlo en la práctica pedagógica se nota un trabajo individual ya que cada docente actúa de acuerdo a su experiencia, dejando al lado el trabajo en equipo y poco refuerzo en las pruebas externas (SABER). Lo cual explica, en cierta medida el bajo desempeño que se evidencia en los resultados de los últimos años.

\section{Referencias}

Allport, G. W. (1973). Psicología de la Personalidad. Revista Interamericana de Psicología, 7, 2-2. https://journal.sipsych.org/index.php/IJP/ article/download/669/568

Barreto, F. y Mejía, B. (2015). La interpretación de la práctica pedagógica de una docente de Matemática. Acta Colombiana de Psicología, (14), 87-96. https://actacolombianapsicologia. ucatolica.edu.co/article/view/426

Castro, E.; Peley, R. y Morillo, R. (2006). La práctica pedagógica y el desarrollo de estrategias instruccionales desde el enfoque constructivista. Revista de Ciencias Sociales, 12(3), 581 - 587. http://dx.doi.org/10.31876/rcs.v12i3.25344

Duarte, L., Ricardo N., y Santos, L. (2018). Dominio afectivo de los estudiantes de educación media hacia las matemáticas. Revista Perspectivas, 3(2), 60-71. https://doi.org/10.22463/25909215.1589

Enamorado, J. (2012). Practicas Pedagógicas de los Docentes en la Enseñanza de la Matemáticas en el I Ciclo en las Escuelas Primarias del Departamento de Ocotepeque (tesis de maestría). Universidad Pedagógica Nacional Francisco Morazán, Tegucigalpa, Honduras. http://www. cervantesvirtual.com/nd/ark:/59851/bmcxw677

Fernández, R., Hernández, C., Prada, R. y Ramírez, P. (2018). Dominio afectivo y prácticas pedagógicas de docentes de Matemáticas: Un estudio de revisión. Revista Espacios, 39(23), 25. https://www.revistaespacios.com/a18v39n23/ a18v39n23p25.pdf 
Fúneme, C., y Hernández, C. (2017). Identificación de procesos matemáticos en la comprensión del concepto de razón en estudiantes universitarios. Ecomatemático, 8(1), 6-14. https://doi. org/10.22463/17948231.1470

Gómez, G. (2010). Las creencias de los profesores de ELE acerca de la práctica de la expresión escrita (memoria de investigación). Universidad de Barcelona, Barcelona, España. http://www. educacionyfp.gob.es/dam/jcr:85b16188f0cd-448f-bdbe-00eaa57b493f/2010-bv-1109gomez-munoz-pdf.pdf

Gómez escobar, A., León, C. y Fernández, R. (2019). Actitudes hacia las Matemáticas y prácticas docentes: un estudio exploratorio en maestros. Revista Perspectivas, 4(1), 23-31. https://doi. org/10.22463/25909215.1752

Jiménez, A., Limas, L. y Alarcón, J. (2016). Prácticas Pedagógicas Matemáticas de Profesores de una Institución Educativa de Enseñanza Básica y Media. Revista Praxis y Saber. 7(13), 127 - 152. https://doi.org/10.19053/22160159.4169

Ministerio de Educación Nacional. (2006). Estándares Básicos de Competencias en Matemáticas. Bogotá: MEN.

Parada, A. (2018). Estrategias Gerenciales para el Reconocimiento del Desempeño Laboral Docente. Mundo FESC, 7(14), 42-56. https:// www.fesc.edu.co/Revistas/OJS/index.php/ mundofesc/article/view/140

Pérez, S. (2000). Modelos de investigación cualitativa. Madrid: Nancea

Ramos Palacios, L.A. \& Casas García, L.M. (2018). Concepciones y creencias de los profesores de Honduras sobre la enseñanza, aprendizaje y evaluación de las matemáticas. Revista Latinoamericana de Investigación en Matemática Educativa, 21(3), 275-299. https:// doi.org/10.12802/relime.18.2132

Rodríguez,E.(2015).El desarrollo dela competencia matemática a través de tareas de Investigación en el aula. Una propuesta de investigación-acción para el primer ciclo de educación primaria (tesis de doctorado). Universidad Nacional de Educación a Distancia, España. http://espacio.uned.es/fez/eserv/tesisuned:EducacionErodriguez/RODRIGUEZ_FRANCISCO_ Encarnacion_Tesis.pdf

Rodríguez, R. y Herrera, M. (2015). Prácticas Pedagógicas de los Docentes de Matemática frente a Estándares y Lineamientos Curriculares en Educación Básica en la Institución Educativa de San Cayetano (proyecto de maestría). Universidad de San Buenaventura, Colombia. http://funes.uniandes.edu.co/10570/1/ Rodriguez2015Practicas.pdf

Rueda, P. y Villaroel, I. (1992). El método Hermenéutico-Dialéctico una estrategia para las ciencias de la conducta. Revista Ciencias de la Educación, 3(5). http://servicio.bc.uc.edu.ve/ educacion/revista/a3n5/3-5-4.pdf

Taylor, S. y Bogdan, R. (1998). Introducción a los métodos cualitativos de investigación. Barcelona: Paidós.

Valiente, S. (2000). Didáctica de la matemática. El libro de los recursos. Madrid: La Muralla.

Vasco, C. (1990). Algunas reflexiones sobre la pedagogía y la didáctica. En: M. Díaz y J. Muñoz (Eds.), Pedagogía, discurso y poder (pp. 107-122). Bogotá: CORPRODIC.

Vázquez, Á. y Manassero, M. (2007). En defensa de las actitudes y emociones en la educación científica. Revista Eureka sobre Enseñanza y Divulgación de las Ciencias. 4(2), 247-271. https://revistas.uca.es/index.php/eureka/article/ view/3803 